\title{
AN EXTENSION OF THE THOMSON PRINCIPLE
}

\author{
A. W. J. STODDART
}

Abstract. The classical Thomson principle, giving lower bounds for the Dirichlet integral, is extended, with modification, to a wider class of test functions. This makes it possible to obtain simpler and better lower bounds.

1. Introduction. Consider a Jordan region $A$ in $m$-dimensional Euclidean space $E_{m}$, with exterior normal $n$ on the boundary $A^{*}$. Let $y$ be a real-valued function and $\psi$ an $m$-vector-valued function on the closure of $A$, each continuous and piecewise continuously differentiable.

The Thomson principle (see [2, p. 43] for a particular version) states that, if $\operatorname{div} \psi=0\left({ }^{*}\right)$, then

$$
\int_{A} y^{\prime 2} d \mu \geqq\left(\int_{A^{*}} y \psi \cdot n d s\right)^{2} / \int_{A} \psi^{2} d \mu
$$

(the second integral is over the boundary hypersurface). This result gives lower bounds for the Dirichlet integral $\int_{A} y^{\prime 2} d \mu$, which can be used, for example, in estimation of capacitance.

In this paper, we shall relax the condition $\left(^{*}\right)$ on $\psi$. This allows us to obtain very simple lower bounds, and to improve particular lower bounds obtained under condition $\left({ }^{*}\right)$.

This work is a by-product of similar work on lower bounds in control systems on multidimensional domains [3], carried out under a Faculty Research Fellowship at Western Michigan University.

2. Modified Thomson principle.

THEOREM. If $I^{*} \operatorname{div} \psi \leqq 0$ and $\left.y\right|_{A^{*}} \geqq 0$, then

$$
\int_{A} y^{\prime 2} d \mu \geqq I^{* 2} / I
$$

where $I=\int_{A} \psi^{2} d \mu, I^{*}=\int_{A^{*} y \psi \cdot n d s .}$

Proof. By the Dirichlet principle,

Received by the editors September 7, 1970 and, in revised form, November 4, 1970. AMS 1970 subject classifications. Primary 26A84; Secondary 31A15.

Key words and phrases. Thomson principle, Dirichlet integral, lower bounds, capacitance. 


$$
\int_{A} y^{\prime 2} d \mu \geqq \int_{A} Y^{\prime 2} d \mu
$$

where $\left.\left.Y\right|_{A^{*}=y}\right|_{A^{*}}$ and $Y$ is harmonic with minimum on $A^{*}$. By the divergence theorem,

$$
\int_{A^{*}} Y \psi \cdot n d s=\int_{A}\left(Y^{\prime} \cdot \psi+Y \operatorname{div} \psi\right) d \mu .
$$

Hence

$$
\begin{aligned}
\int_{A} Y^{\prime 2} d \mu & =\int_{A}\left(Y^{\prime 2}+Y^{\prime} \cdot \psi+Y \operatorname{div} \psi\right) d \mu-\int_{A^{*}} Y \psi \cdot n d s \\
& \geqq L(\psi)=-\frac{1}{4} \int_{A} \psi^{2} d \mu-\int_{A^{*}} y \psi \cdot n d s
\end{aligned}
$$

provided $\operatorname{div} \psi \geqq 0$. Now

$$
\begin{aligned}
L(t \psi) & =-\frac{1}{4} t^{2} I-t I^{*}=-I\left(\frac{1}{2} t+I^{*} / I\right)^{2}+I^{* 2} / I \\
& =I^{* 2} / I \quad \text { when } t=-2 I^{*} / I,
\end{aligned}
$$

so $\int_{A} y^{\prime 2} d \mu \geqq I^{* 2} / I$ provided $\frac{1}{2} I \operatorname{div} t \psi=-I^{*} \operatorname{div} \psi \geqq 0$.

REMARK 1 . If $\operatorname{div} \psi=0$, the condition $y \mid A^{*} \geqq 0$ is unnecessary, and we get the classical Thomson principle.

REMARK 2. We can extend our result to a combination of linearly independent functions $\psi_{i}$, with possible improvement of the lower bound. In $L(\psi)=-\frac{1}{4} \int_{A} \psi^{2} d \mu-\int_{A^{*}} y \psi \cdot n d s$, put $\psi=t_{i} \psi_{i}$ (with repeated subscripts indicating summation). With

$$
\begin{gathered}
I_{i j}=\int_{A} \psi_{i} \cdot \psi_{j} d \mu, \quad J=\left[I_{i j}\right]^{-1}, \quad \text { and } I_{i}^{*}=\int_{A^{*}} y \psi_{i} \cdot n d s \\
\begin{aligned}
L(\psi) & =-\frac{1}{4} t_{i} I_{i j} t_{j}-t_{i} I_{i}^{*} \\
& =-\left(\frac{1}{2} t_{i}+I_{h}^{*} J_{h i}\right) I_{i j}\left(\frac{1}{2} t_{j}+J_{j k} I_{k}^{*}\right)+I_{j}^{*} J_{j k} I_{k}^{*} \\
& =I_{j}^{*} J_{j k} I_{k}^{*} \quad \text { when } t_{j}=-2 J_{j k} I_{k}^{*} .
\end{aligned}
\end{gathered}
$$

Note that the matrix $\left[I_{i j}\right]$ is nonsingular since $t_{i} I_{i j} t_{j}=\int_{A}\left(t_{i} \psi_{i}\right)^{2} d \mu>0$ if $\left[t_{i}\right] \neq 0$. The condition on $\psi$ now reads $\operatorname{div} \psi_{j} J_{j k} I_{k}^{*} \leqq 0$.

3. Estimation of capacitance. Let $A$ be the region exterior to a closed hypersurface $A_{1}^{*}$ and interior to a second closed hypersurface $A_{0}^{*}$. We can calculate the capacitance of such regions by minimizing

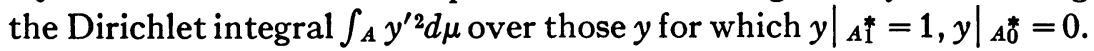

To obtain a lower bound, take $\psi_{i}$ with components $\left(\psi_{i}\right)_{k}=0$ for $k \neq i, x_{i}$ for $k=i$. The $\psi_{i}$ are obviously independent. Then $I_{i j}=0$ for 
$i \neq j, \int_{\Lambda} x_{i}{ }^{2} d \mu$ for $i=j$; and $I_{i}^{*}=-\mu_{1}$ where $\mu_{1}$ is the measure of the region $A_{1}$ enclosed by $A_{1}^{*}$. Here

$$
\operatorname{div} \psi_{j} J_{j k} I_{k}^{*}=-\mu_{1} \sum 1 / \int_{A} x_{i}^{2} d \mu<0 .
$$

Thus we have the crude but simple lower bound

$$
\mu_{1}^{2} \sum 1 / \int_{\Delta} x_{i}^{2} d \mu .
$$

Coordinate axes should be chosen to make this as large as possible, that is, along the principal axes of the positive matrix $\left[\int_{A} x_{i} x_{j} d \mu\right]$, with origin at the centroid of $A$.

As another choice for $\psi$, consider $\psi=r^{k} x$ where $r=|x|, 0 \in A_{1}$. Then $I=\int_{A} r^{2 k+2} d \mu$

$$
\begin{array}{rlrl}
I^{*} & =-(m+k) \int_{\Delta_{1}} r^{k} d \mu & & \text { for } k>-m, \\
& =-s_{m} & \text { for } k=-m,
\end{array}
$$

where $s_{m}$ is the $(m-1)$-dimensional measure of the unit sphere in $E_{m}$; and $\operatorname{div} \psi=(m+k) r^{k}$, so $\operatorname{div} \psi I^{*} \leqq 0$ for $k \geqq-m$. Thus, for $k>-m$, we have the lower bound

$$
L_{k}=(m+k)^{2}\left(\int_{A_{1}}{ }^{k} d \mu\right)^{2} / \int_{\Delta}{ }^{2 k+2} d \mu .
$$

4. An example. Let $A$ in $E_{8}$ be the ellipsoidal shell

$$
\left\{x: a \leqq r\left(c^{2} \sin ^{2} \phi+1\right)^{1 / 2} \leqq b\right\}
$$

where $\phi$ is the latitudinal coordinate. Consider $\int_{A} y^{\prime 2} d \mu$ for $y=1$ on the inner boundary and $y=0$ on the outer boundary. Here

$$
\begin{aligned}
& \int_{A_{1}} r^{k} d \mu=4 \pi K_{(k+3) / 2} a^{k+3} /(k+3), \\
& \int_{A} r^{2 k+2} d \mu=4 \pi K_{(2 k+5) / 2}\left(b^{2 k+5}-a^{2 k+5}\right) /(2 k+5)
\end{aligned}
$$

where $K_{p}=\int_{0}^{1}\left(c^{2} x^{2}+1\right)^{-p} d x$, for which we have the recurrence relation

$$
K_{p}=K_{p-1}(2 p-3) /(2 p-2)+\left(1+c^{2}\right)^{1-p} /(2 p-2)
$$

with $K_{0}=1, K_{1 / 2}=\left(\sinh ^{-1} c\right) / c$. 
By $\S 3$, we have the lower bounds

$$
L_{k}=4 \pi K_{(k+3) / 2}^{2}(2 k+5) a^{2 k+6} / K_{(2 k+5) / 2}\left(b^{2 k+5}-a^{2 k+i}\right)
$$

for $k>-3$. For the case $k=-3$ in which $\operatorname{div} \psi=0$,

$$
L_{-3}=8 \pi a b /(b-a)\left[\left(1+c^{2}\right)^{1 / 2}+\left(\sinh ^{-1} c\right) / c\right]
$$

so $c L_{-3} \rightarrow 8 \pi a b /(b-a)$ as $c \rightarrow \infty$. For the case $k=-2$ in which $\operatorname{div} \psi \neq 0$,

$$
L_{-2}=4 \pi a^{2}\left(\sinh ^{-1} c\right) /(b-a) c
$$

so $c L_{-2} \rightarrow \infty$ as $c \rightarrow \infty$. Thus for large $c, k=-2$ gives a better lower bound than the classical $k=-3$.

\section{REFERENCES}

1. L. E. Payne, Isoperimetric inequalities and their applications, SIAM Rev. 9 (1967), 453-488. MR 36 \#2058.

2. G. Polya and G. Szegö, Isoperimetric inequalities in mathematical physics, Ann. of Math. Studies, no. 27, Princeton Univ. Press, Princeton, N.J., 1951. MR 13, 270.

3. A. W. J. Stoddart, Estimation of optimality for multidimensional control systems, J. Optimization Theory Appl. 3 (1969), 385-391. MR 39 \#7984.

Western Michigan University, Kalamazoo, Michigan 49001 